\title{
Tumor Identifier Accepted Record Flag
}

National Cancer Institute

\section{Source}

National Cancer Institute. Tumor Identifier Accepted Record Flag. NCI Thesaurus. Code C117428.

An indication or description of a record that is considered to be the accepted and final evaluation for the tumor identification. 